\title{
Influence of the drying method on the sorption properties the biomass of Chlorella sorokiniana microalgae
}

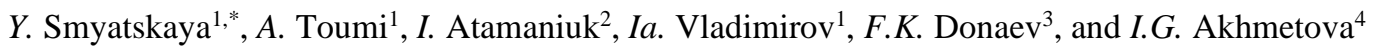 \\ ${ }^{1}$ Peter the Great St. Petersburg Polytechnic University, Saint-Petersburg, Russia \\ ${ }^{2}$ Hamburg University of Technology, Hamburg, Germany \\ ${ }^{3}$ Tajik Technical University Named after Academic M.S.Osimi, Department of Power Engineering, Dushanbe, Tajikistan \\ ${ }^{4}$ Kazan State Power Engineering University, Kazan, Russia
}

\begin{abstract}
In this paper, it is proposed to use the biomass of microalgae Chlorella sorokiniana as a biosorbent for wastewater treatment, as well as an oral sorbent. Biosorbents are capable of adsorbing both organic and inorganic compounds, including heavy metals. The sorption capacity depends on the type of aquatic plant and microalgae strain. The use of microalgae and aquatic plants as biosorbents for pollutant treatments is discussed in the introduction part. The biomass of microalgae Chlorella sorokiniana was chosen as the object of this study. The cultivation conditions (temperature, light, $\mathrm{pH}$ and aeration) and the optimal biomass harvesting parameters are presented. Dehydration of biomass was carried out in two ways: IR-drying and freeze-drying. The obtained samples were tested for the ability of the biomass to extract heavy metal ions (zinc, cadmium, zinc, copper) from standard solutions. The initial concentration of heavy metal ions in the working solutions was $10 \mathrm{mg} / \mathrm{l}$. Results show that the lyophilized samples demonstrated up to $99.9 \%$ of heavy metal removal efficiency. The paper also presents the composition of Chlorella sorokiniana biomass, in which up to $40.97-41.87 \%$ are proteins. The analysis of the amino-acid composition showed a ratio of essential to non-essential amino-acids higher than 0.8 . All the above results confirm the possibility of using microalgae biomass as an oral sorbent and as an additive in the production of functional foods.
\end{abstract}

\section{Introduction}

Marine plants are widely used to remove heavy metal ions of zinc and copper from water [1]. Although it is possible to use living algae for water depuration, it is most commonly proposed to use dry algae as a sorbent. The cell-walls of algae cells contain mainly cellulose and proteins combined to polysaccharides. Cell-walls is a key factor in the removal of heavy metals from water using algae. The composition of the cell-wall varies depending on the algae species; therefore, the ability to bind metal ions also differs. Algal cell-walls are rich in functional groups such as carboxyl, amine, hydroxyl, phosphate, imidazole and sulfate [2]. These groups are negatively charged and attract positive metal ions. However, at low $\mathrm{pH}$, they are partially protonated lowering their sorption capacity towards positively charged ions. In a previous study, Chlorella sorokiniana biomass was grown in cultivators, then dried in a drying oven and used to extract gallium ions. The sorption capacity reached $38.5 \mathrm{mg} / \mathrm{g}$ [3]. Another study investigated the biosorption of lead, cadmium, copper, and arsenic ions using algae [4]. The biosorption of these metals is based on an ion-exchange mechanism, accompanied by the release of metals, such as calcium, magnesium and sodium. The $\mathrm{pH}$ value, initial concentrations and temperature of extraction were also studied and the optimal sorption conditions were selected. To improve the sorption properties, it is proposed to modify the sorption materials [5]. In Jeyakumar et al. 2014, the marine green algae Ulva Fasciata was used to prepare activated carbon for the study of the adsorption of lead (II) ions [6]. The authors proposed to impregnate the dried algae in chloride salts, such as calcium chloride, for 24 hours. The biomass was well impregnated in 5 liters of a $10 \%$ solution of calcium chloride. After 24 hours, the excess solution was decanted and air dried. Then the material was carbonized at $400{ }^{\circ} \mathrm{C}$. The dried samples were crushed and activated in a muffle furnace, kept at $800^{\circ} \mathrm{C}$ for 10 minutes. After activation, the carbon was washed with $\mathrm{HCl} 4 \mathrm{~N}$ to remove cations. The materials was abundantly washed with water to remove excess acid, then dried and ground to a powder.

Aquatic weeds can also be used to extract heavy metal ions [7]. Activated carbon obtained from Eichhornia was used as an adsorbent to remove lead from aqueous solutions. Its production presents the advantage of being cost-efficient. Several parameters such as the activation time, ion concentration, adsorbent

*Corresponding author: politaevana1971@gmail.com 
dose and $\mathrm{pH}$ have been examined. Sorption equilibrium was achieved after 100 minutes in the solution containing $15 \mathrm{mg} / \mathrm{l}$ and after 125 minutes in the solution containing $20 \mathrm{mg} / \mathrm{l}$ and $25 \mathrm{mg} / \mathrm{l}$ of lead ions, respectively. The adsorption parameters were determined using Langmuir and Freundlich isotherm models.

Over the last few years, many inexpensive sorbents have been investigated [8-9]. Brown algae have proven to be the most effective and promising in this regard [10]. Biosorption has been proven to effectively remove heavy metals such as $\mathrm{Cd} 2+, \mathrm{Cu} 2+, \mathrm{Zn} 2+, \mathrm{Pb} 2+, \mathrm{Cr} 3+$ and $\mathrm{Hg} 2+$.

Studies of duckweed Lemna minor demonstrated that arsenic absorption can be carried out without prior treatment [11]. Various arsenic adsorption / desorption parameters have been studied. The maximum sorption of arsenic was obtained under the following conditions: an initial arsenic concentration of $100 \mu \mathrm{g} / \mathrm{l}$, using $3 \mathrm{~g}$ of duckweed. The average particle size represented $0.595 \mathrm{~mm}$, the flow rate: $1.67 \mathrm{ml} / \mathrm{min}$ and the $\mathrm{pH}$ reached 5.5. Desorption was carried out using a $1 \mathrm{M}$ solution of sulfuric acid. The results showed a degree of desorption of $97.67 \%$.

Another work was devoted to study the sorption capacity of Turbinaria turbinata, a brown alga widely available in many tropical areas; for removing hexavalent chromium from aqueous media [12]. The dried alga has several polyfunctional metal binding sites, which allows its use as a sorbent. Surface modification (pretreatment with acid) and structural modification (preparation of chemically and physically activated carbons) also improve its adsorption properties. Depending on the experimental conditions, a significant improvement in metal sorption was obtained (up to 40 times higher).

Due to their unique sorption properties, microalgae biomass should be investigated not only for water purification, but also for its potential detoxifying effects on the human body.

To obtain biosorbent with the desired properties and a biomass with a high growth rate, it is necessary to select the optimal cultivation conditions. A complete review of such literature is given in the articles [13-15] in which the cultivation conditions are fully described: the composition of the cultivation medium, the shape of the cultivator, the illumination, the temperature, the carbon dioxide concentration and aeration. A relevant work of Dagestan scientists proposes to cultivate microalgae on geothermal waters. A certain number of hot springs can be found on the territory of Dagestan. These can be used as a source of energy, raw material and for their balneological value. The geothermal waters, which contain biogenic elements in sufficient quantity, are described as a favorable medium for the cultivation of chlorella. The work also presents data on the ability of microalgae to adsorb excess concentrations of phenol to safe values [16].

Concas et al. built a mathematical model on the basis of experimental date that will allow, in the future, to predict the response of organisms in variable conditions of growth [17]. The growth and lipid accumulation patterns of Chlorella sorokiniana grown in periodic culture and photobioreactors BIOCOIL have been studied. The collected data have been used for modeling and building an optimal mathematical model. The comparison of the results obtained with both the model and the experimental data showed a good concordance. Thus, it has been proven that it is possible to simulate the growth of microalgae, which allows to develop suitable strategies for choosing more optimal performance parameters of photobioreactors.

After the cultivation process follows the biomass concentration step. There are various ways of harvesting microalgae biomass: the addition of chemical reagents (flocculants, coagulants), microfiltration, centrifugation, ultrasonic separation etc. The harvesting process can proceed in one or several stages.

Given the rich composition of Chlorella microalgae, it is widely used as a biological food additive and in the production of functional foods. The composition of the Chlorella includes polyunsaturated fatty acids, proteins, minerals, fibers, chlorophylls, vitamins (C, betacarotene, B1, B2, B6, B12, niacin, pantothenic acid, folic acid, choline, vitamin $\mathrm{E}$ and vitamin $\mathrm{K}$ ). This microalga also contains carotenoids, flavonoids, polyphenols, polysaccharides and glycoproteins.

\section{Materials and methods}

In this study, it is proposed to use the biomass of microalgae Chlorella sorokiniana as a biosorbent. The biomass was grown in cultivators using a nutrient medium with a balanced composition of macro- and microelements. The optimal conditions for the cultivation of the biomass of Chlorella sorokiniana are presented in the following works [18-21]. The biomass was grown using fluorescent lamps for illumination, a constant aeration at a rate of $1.5 \mathrm{l} / \mathrm{min}$ and a temperature of $25^{\circ} \mathrm{C}$. The cultivation lasted 10 days reaching an optical density of 1.7 , which corresponds to 43 million cells $/ \mathrm{ml}$.

The cultivated biomass was then concentrated using a PE 6900 centrifuge at a speed of $6000 \mathrm{rpm}$ for 10 minutes. Centrifugation is similar to sedimentation, in which the gravitational force is replaced by centrifugal acceleration to increase the concentration of solid particles. Particle size and density difference are key factors in centrifugal separation. The microalgae concentrate can be then obtained by simply draining the supernatant. This type of harvesting is usually recommended for the production of high-value metabolites or as a second stage dehydration method for concentrating algae suspensions from $1-5 \%$ solids to $15 \%$ and above.

Then, 2 types of samples were prepared:

- Biomass of Chlorella sorokiniana microalgae dried by Infrared (IR) drying;

- Biomass of the microalga Chlorella sorokiniana dried by freeze-drying using the lyophilizer AK-50N (Proflab). For this purpose, the sample had to be completely frozen before hands at a temperature of 
$-180^{\circ} \mathrm{C}$ and only then subjected to freeze-drying under the following conditions: pressure $-0.5 \mathrm{~atm}$, time -24 hours, temperature of the condenser $-500^{\circ} \mathrm{C}$.

To determine the efficiency of the removal of heavy metals by biosorption, working solutions were prepared from standard samples containing zinc, cadmium, lead and copper ions with a concentration of $10 \mathrm{mg} / \mathrm{l}$. Each sorbent in the amount of $100 \mathrm{~g}$ was added to $1000 \mathrm{ml}$ of the working solution containing the four heavy metal ions. The sorption process was carried out by first, a shaking step using a laboratory platform shaker for 2 hours. The mixtures were then left at rest for 22 hours. After that, the biosorbent was separated from the solution and the residual concentrations of metals were determined by the voltammetry (Russian Federal Environmental Regulatory Document or PND F 14.1: 2: 4.222-06) on the TA-Lab device. This analysis presents a $25 \%$ measurement accuracy.

The sorption capacity was calculated according to the formula (1). The results are presented in table 1 .

$$
q=\frac{C_{i}-C_{f i n}}{m} \times V
$$

where $q$ is the sorption capacity; $C i$ and $C_{f i n}$ are the initial and final concentrations of heavy metals of the solution; $V(L)$ is the volume of solution; $m(g)$ is the waste amount.

The cleaning efficiency was determined by the following formula:

$$
E=\frac{C_{i}-C_{f i n}}{C_{i}} \times 100 \%
$$

where $C i$ is the initial concentration, $\mathrm{mg} / \mathrm{l} ; C_{f i n}$ is the final concentration, $\mathrm{mg} / \mathrm{l}$.

For the microstructural study, a Zeiss Supra 55 VP FEG-SEM microscope was used.

\section{Results and discussion}

The development of natural oral sorbents is a promising and relevant task. The biomass of the microalgae Chlorella sorokiniana has the ability to adsorb heavy metals, such as lead, cadmium, zinc and copper. Table 1 presents average results of two parallel experiments of the removal of heavy metals from the working solutions.

The microalgae biomass has rather high sorption characteristics. The cleaning efficiency reached up to $98 \%$ for lead ions for the sample after IR drying. Drying the sample under by lyophilization allows obtaining a sample with a developed porous structure (Figure 1). Another advantage of lyophilization is to obtain a partially disinfected sample due to freezing of biomass at low temperatures, which is relevant when using biomass as a natural oral sorbent.

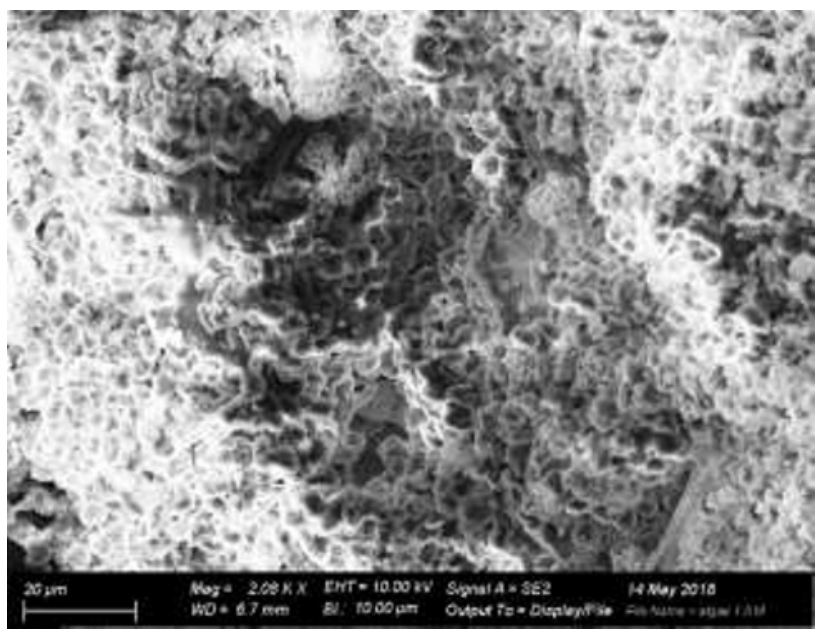

Fig. 1. Microscopic image of the biomass sample of Chlorella sorokiniana microalgae after freeze drying.

Microscopic examination of Chlorella sorokiniana sample showed that after freeze drying, the cells retain their shape and form uniform layers. The size of cells is approximately 3 to 5 microns.

The removal efficiency of heavy metals (zinc, cadmium, lead, copper) by the lyophilized biomass reached $9,9 \%$. Sorption could be attributed to physical or chemical processes and further research is required to define the exact sorption mechanisms.

It is known that microalgae have a rich composition of valuable elements. The composition of the microalgae Chlorella sorokiniana was determined using various analytical methods at Hamburg University of Technology (TUHH). The overall composition of the main structural components of Chlorella sorokiniana,

Table 1. Cleaning efficiency and sorption capacity of the biomass of microalgae Chlorella sorokiniana after IR-drying and freeze-drying.

\begin{tabular}{|c|c|c|c|}
\hline & C fin, mg/l & E, \% & q, mg/g \\
\hline Adsorbate & Biomass after IR-drying & 0.059 \\
\hline Zinc & 4,1 & 59 & 0.089 \\
\hline Cadmium & 1.1 & 89 & 0.098 \\
\hline Lead & 0.2 & 98 & 0.089 \\
\hline Copper & 0,5 & 89 & 0.098 \\
\hline \multicolumn{2}{|c|}{ Biomass after freeze-drying } \\
\hline Zinc & 0,165 & 98,35 & 0.098 \\
\hline Cadmium & 0.10 & 98,95 & 0.099 \\
\hline Lead & 0.037 & 99,62 & 0.099 \\
\hline
\end{tabular}


grown in two closed cultivators (M1 and M2), is presented in Figure 2. Due to the large number of samples, the results are presented as average values.

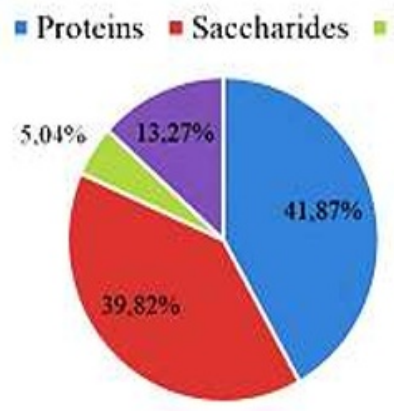

M1

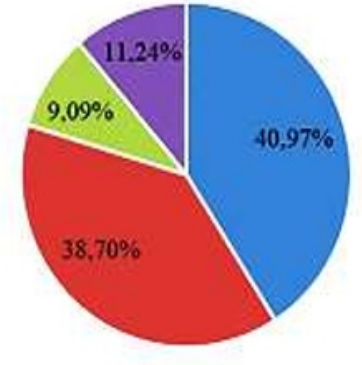

M2
Fig. 2. The total chemical composition of Chlorella sorokiniana from cultivators M1 and M2.

As seen in Figure 2, the main components of Chlorella sorokiniana are proteins (40.97-41.87\%), carbohydrates (38.70-39.42\%), lipids (11.24-13.27\%), pigments and inorganic substances (5.04-9.09\%). Microalgae biomass is a rich source of vegetable protein. The amino acid composition of Chlorella sorokiniana and the ratio of essential to non-essential amino acids are presented in Figures 3,4.

Figure 3 shows that 18 essential and non-essential amino acids have been identified in the microalgal biomass.
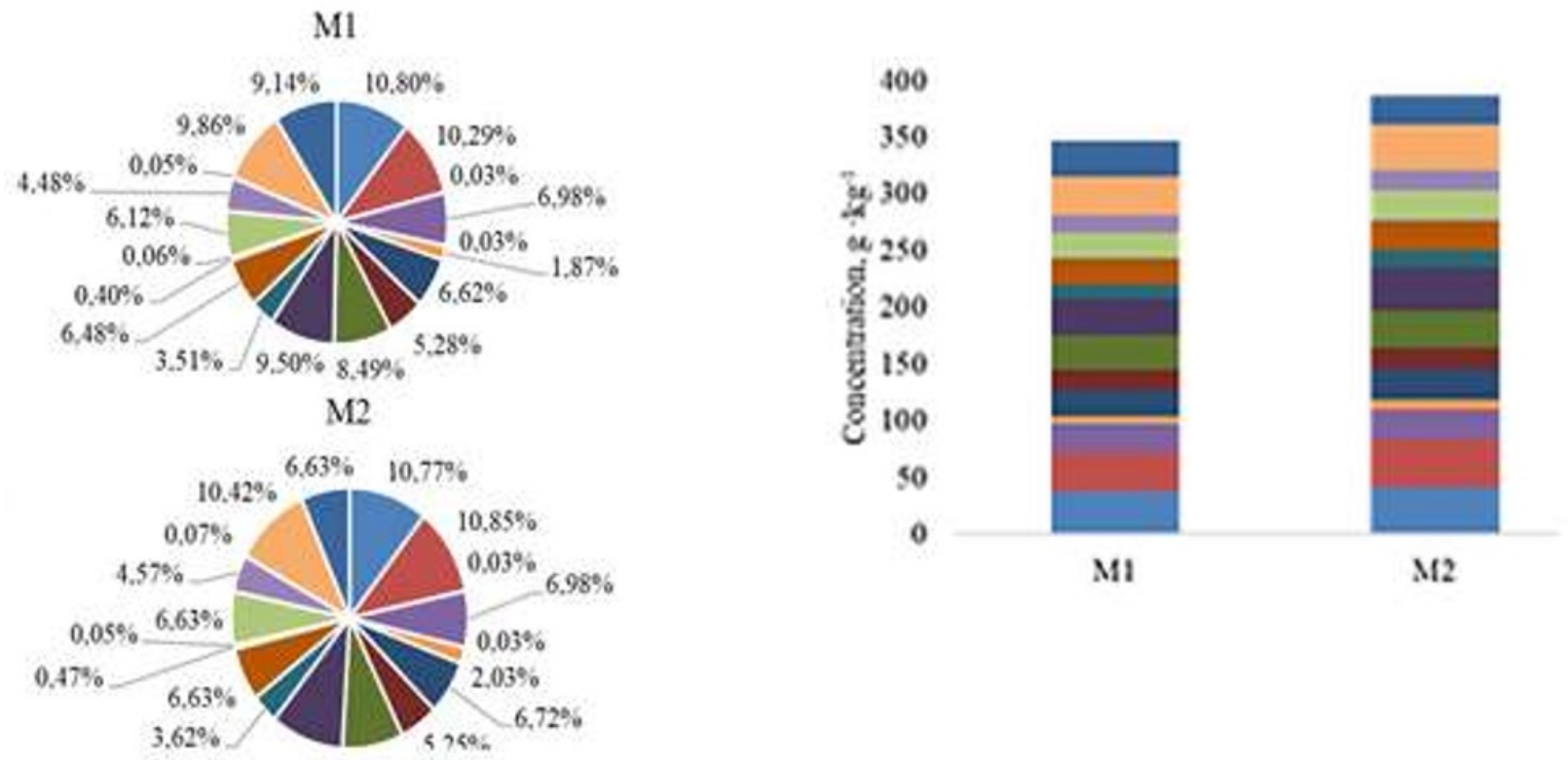

Fig. 4. The ratio of essential to non-essential amino-acids.

In both cases, the ratio of essential to non-essential amino acids was higher than 0.8 (Figure 4), and, if compared to the recommendations of the use of essential amino acids in food given by the World Health Organization, this microalga could be used in the food industry.

Proper nutrition ensures the proper growth and development of children, contributes to the prevention of diseases, increases efficiency and prolongs life, while unbalanced diets, on the contrary, may lead to the emergence of many health issues. Russian scientists point out that often, there is a chronic lack of protein in diet, especially in childhood and old age [22]. This fact is the cause of an increase in the occurrence of alimentary-dependent diseases already in childhood and adolescence such as: rickets and hypotrophy by 2 times, anemia by 1.5 times and endocrine pathology by 1.5 times. Over the past 10 years, the prevalence of diseases

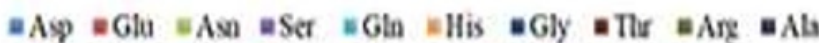

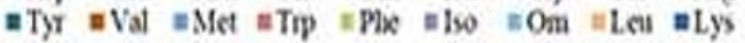

Fig. 3. The amino acid composition of the biomass of microalgae Chlorella sorokiniana. 
of the digestive system among schoolchildren has increased by 3.3 times, which is also directly related to nutritional deficiency.

Additives containing Chlorella sorokiniana microalgae could be used to enrich food products with vegetal protein. Further investigations are required for studying the potential use of this microalgae as an oral sorbent in the case of a heavy metal acute intoxication. For people who do not consume animal products, chlorella could be proposed a solution to the problem of a protein deficient diet. The development of microalgaebased food products could higher the nutritional value of functional foods as this organism contains high amounts of protein as well as polyunsaturated fatty acids (Omega3-6-9) and thus, could be recommended to enrich one's diet with essential nutrients. However, a rigorous quality control of dietary products based on microalgae is still to be developed.

\section{Conclusions}

1. The sorption properties of the biomass samples of Chlorella sorokiniana microalgae after IR drying and lyophilization were studied. A 98 to $99 \%$ sorption capacity could be achieved in regards to cadmium, lead, copper and zinc ions when using the freeze-dried biomass as a biosorbent.

2. The total biomass composition of Chlorella sorokiniana was studied, which showed the presence of proteins (40.97-41.87\%), carbohydrates (38.70$39.42 \%$ ), lipids (11.24-13.27\%), pigments and inorganic substances (5.04-9.09\%).

3. The study on the amino acid composition showed the presence of 18 essential and non-essential amino acids and a ratio of essential to non-essential amino acids higher than 0.8 .

4. The sorption properties and biological value of the biomass of microalgae Chlorella sorokiniana allows it to be used as oral sorbent and a food additive.

\section{References}

[1] L. C. Ajjabi, L. Chouba, Biosorption of Cu2+ and $\mathrm{Zn} 2+$ from aqueous solutions by dried marine green macroalga Chaetomorpha linum. Journal of Environmental Management, 90, 3485-489 (2009)

[2] V. Javanbakht, S. A. Alavi, H. Zilouei, Mechanisms of heavy metal removal using microorganisms as biosorbent, Water Science \& Technology 69(9), 1775-1787 (2014)

[3] Li. Mengling, et al. Algae based sorbents for removal of gallium from semiconductor manufacturing wastewater. Clean Technologies and Environmental Policy. 20, 899-907 (2018)

[4] A.H. Sulaymon, et al. (2013) Competitive biosorption of lead, cadmium, copper, and arsenic ions using algae. Environ Sci Pollut Res 20(5), 3011-23. https ://doi. org/10.1007/s1135 6-012-1208-2.

[5] M. Nadeem, M. Shabbir, M.A. Abdullah, Sorption of cadmium from aqueous solution by surfactant-modified carbon adsorbents. Chem. Eng. 148, 365-70 (2009)

[6] R. Jeyakumar, P. Suresh, and V. Chandrasekaran, Adsorption of lead(II) ions by activated carbons prepared from marine green algae: Equilibrium and kinetics studies. Int. J. of Industrial Chemistry (Springer) 5, 10 (2014)

[7] P. Shekinah, K. Kadirvelu, P. Kanmani, P. Senthilkumar, V. Subburam Adsorption of lead(II) from aqueous solution by activated carbon prepared from Eichhornia //Journal of Chemical Technology \& Biotechnology. 77(4), 458-464

[8] N.A. Politaeva, V.V. Slugin, E.A. Taranovskaya, M.A. Soloviev, A.M. Zakharevich, Granulated sorption materials for waste waters purufucation from zink ions (Zn2+) Izvestiya Vysshikh Uchebnykh Zavedenii, Seriya Khimiya i Khimicheskaya 60(7), 85-90 (2017)

[9] L.N. Ol'shanskaya, N.A. Sobgaida, Yu.A. Tarushkina, A.V. Stoyanov, Effect of a magnetic field on extraction of heavy metals from waste water with duckweed Chemical and Petroleum Engineering-44(7-8), 475-479 (2008)

[10] T. A. Davis, B. Volesky, A. Mucci, A review of the biochemistry of heavy metal biosorption by brown algae// Water Research. 37(18), November, 4311-4330 (2003)

[11] R. Tasrina, T. Choudhury, M. N. Acher, S. B. Amin, Quraishiland A. I. Mustafa Removal of Arsenic (III) from Groundwater by Adsorption onto Duckweed (Lemna minor)//International Research Journal of Pure \&Applied Chemistry 6(3), 120-127, (2015)

[12] C. Yacou, et al. Chemical structure investigation of tropical Turbinaria turbinata seaweeds and its derived carbon sorbents applied for the removal of hexavalent chromium in water //Algae esearch 34, 25$36(2018)$

[13] S. Daliry, A. Hallajisani, J. M. Roshandeh, et al. Investigation of optimal condition for Chlorella vulgaris microalgae growth. Global Journal of Environmental Science and Management. 3(2), 217-230 (2017)

[14] M.C. Franco, M. F. Buffing, M. Janssen et al Performance of Chlorella sorokiniana under simulated extreme winter conditions // Journal of Applied Phycology DOI 10.1007/s10811-011-9687-y. (2011)

[15] S. Negi, A. N. Barry, N. Friedland et al. Impact of nitrogen limitation on biomass, photosynthesis, and lipid accumulation in Chlorella sorokiniana [Text] Journal of Applied Phycology. DOI 10.1007/s10811-015-0652-z. (2015)

[16] I.M. Abdulagatov, A.B. Alkhasov, G.D. Dogeev, N.R. Tumalaev, R. Aliev, G.B. Badavov, A.M. Aliyev, A.S. Salikhova, Microalgae and their technological applications in energy and environmental protection // South of Russia: ecology, development. 13(1), 66-183 DOI: 10.18470 / 1992-1098-2018-1-166$183(2018)$

[17] A. Concas, V. Malavasib, M. Pisua, et al. Experiments and Modeling of the Growth of $\mathrm{C}$. sorokiniana in Lab Batch and BIOCOIL 
Photobioreactors for Lipid production [Text] Chemical engineering transactions. 57, 1-6(2017)

[18] N.A. Politaeva, T.A. Kuznetsova, Y.A. Smyatskaya, E.V. Trukhina, I. Atamanyuk, Energy Production from Chlorella Algae Biomass Under St. Petersburg Climatic Conditions , Chemical and Petroleum Engineering 53(11-12), 801-805 (2018)

[19] N. Politaeva, T. Kuznetsova, Y. Smyatskaya, I. Atamaniuk, E. Trukhina, Chlorella Microalga Biomass Cultivation for Obtaining Energy in Climatic Conditions of St. Petersburg, Advances in Intelligent Systems and Computing, 692, 555-562 (2018)

[20] N. Politaeva, T. Kuznetsova, Y. Smyatskaya, E. Trukhina, F. Ovchinnikov, Impact of various physical exposures on Chlorella Sorokiniana microalgae cultivation International Journal of Applied Engineering Research 12(21), 11488-11492 (2017)

[21] N. Politaeva, Y. Smyatskaya, V. Slugin, A. Toumi, M.Bouabdelli. Effect of laser radiation on the cultivation rate of the microalga Chlorella sorokiniana as a source of biofuel// IOP Conference Series: Earth and Environmental Science. 012001, 115 (2018)

[22] E.T. Zuev, Functional drinks: their place in the concept of a healthy diet. Nutrition and Health journal, 90-95 (2004) 